QUARTERLY OF APPLIED MATHEMATICS

VOLUME LXIII, NUMBER 1

MARCH 2005, PAGES 1-12

S $0033-569 X(04) 00943-7$

Article electronically published on December 14, 2004

\title{
EXISTENCE AND NON-EXISTENCE OF SOLUTIONS TO THE GINZBURG-LANDAU EQUATIONS IN A SEMI-INFINITE SUPERCONDUCTING FILM
}

\author{
BY \\ Y. ALMOG \\ Faculty of Mathematics, Technion - Israel Institute of Technology, Haifa 32000, Israel
}

Abstract. For the problem

$$
\begin{gathered}
\frac{\psi^{\prime \prime}}{\kappa^{2}}=\psi^{3}-\psi+A^{2} \psi, \\
A^{\prime \prime}=\psi^{2} A, \\
\psi^{\prime}(0)=\psi(\infty)=0, \\
A^{\prime}(0)=A^{\prime}(\infty)=h,
\end{gathered}
$$

it is proved for type II superconductors $(\kappa>1 / \sqrt{2})$ that

(1) No solutions can exist for $h \leq 1 / \sqrt{2}$ other than the normal state $\psi \equiv 0, A=$ $h x+C$

(2) Positive solutions $(\psi>0)$ exist whenever $1 / \sqrt{2}<h<h_{c_{3}} \approx 1.7 \kappa$;

(3) As $h \downarrow 1 / \sqrt{2}$, the limit of any converging subsequence satisfies $A=0, \psi=1$ at infinity.

1. Introduction. When a superconducting body is placed under the action of an applied magnetic field, the material would revert to its normal state for sufficiently strong applied field. Linear bifurcation analysis of the normal state [15, 5] shows that if the applied magnetic field $h$ is lowered below a certain critical field, which has been termed $h_{c_{3}}$ or the onset field, the material becomes superconducting once again. When $h \approx h_{c_{3}}$ it is well known that the superconducting region is concentrated in a narrow layer near the boundaries [15, 5. If the field is lowered further, the superconducting region is expected to spread from the boundaries into the domain's interior [8, 16].

The first case in which the bifurcation from the normal state to the superconducting one, in the presence of boundaries, was calculated is the case of a half-plane [15]. The significance of Saint-James and de Gennes' solution [15] extends far beyond the simple, one-dimensional example of a half-plane. It was proved, first for films [7], then for disks [4], and finally for general two-dimensional domains with smooth boundaries [14 12],

Received January 21, 2003.

2000 Mathematics Subject Classification. Primary 82D55. 
that as the domain's scale tends to infinity, the onset field tends to de-Gennes' value. Furthermore, the solution in the boundary layer can be approximated by the solution to the one-dimensional problem.

Extrapolating into the non-linear regime, it appears reasonable to believe that the one-dimensional non-linear solution can serve as a good approximation for the behaviour of the solution near the boundaries, at least as long as $h_{0}<h<h_{c_{3}}$ for some positive $h_{0}$. This, among other things, has been the motivation for the careful and extensive numerical study of the one-dimensional problem in 2 in finite intervals.

Consider then a semi-infinite film of superconducting material. The Ginzburg-Landau equations may be written in their one-dimensional form [9].

$$
\begin{gathered}
\frac{\psi^{\prime \prime}}{\kappa^{2}}=\psi^{3}-\psi+A^{2} \psi, \\
A^{\prime \prime}=\psi^{2} A
\end{gathered}
$$

where $\psi$ is the superconducting order parameter (the gauge has been chosen so that $\psi$ would be real), $\boldsymbol{A}=A \hat{\boldsymbol{y}}$ is the magnetic vector potential, the magnetic field is given by $\boldsymbol{H}=A^{\prime} \hat{\boldsymbol{y}}$, and $\kappa$ is the Ginzburg-Landau parameter. The boundary conditions satisfied by $\psi$ and $A$ are

$$
\begin{aligned}
\psi^{\prime}(0) & =\lim _{x \rightarrow \infty} \psi=0, \\
A^{\prime}(0) & =\lim _{x \rightarrow \infty} A^{\prime}=h .
\end{aligned}
$$

In the present contribution we focus on the above problem for type II superconductors $(\kappa>1 / \sqrt{2})$. We prove the following results:

(1) Positive solutions $(\psi>0)$ exist whenever $1 / \sqrt{2}<h<h_{c_{3}} \approx 1.7 \kappa$.

(2) No positive solutions can exist for $h \leq 1 / \sqrt{2}$. This does not mean that the normal state would prevail, since it is unstable, but rather that the solution of the time dependent Ginzburg-Landau equation satisfying $[1.1 \mathrm{H}, \mathrm{f})$ must be timedependent.

(3) As $h \downarrow 1 / \sqrt{2}$ the limit of any converging subsequence satisfies $A=0, \psi=1$ at infinity.

We also discuss the behaviour of solutions in the case $h-1 / \sqrt{2} \ll 1$. The above results are in agreement with conjecture 4.8 in [2].

In the next section we prove the existence result, in $\S 3$ we prove their non-existence for $h \leq 1 / \sqrt{2}$, and in $\S 4$ we discuss the limit $h \downarrow 1 / \sqrt{2}$. In the last section we briefly summarize the work and address some key points, insufficiently emphasized within the analysis.

2. Existence. We define the onset field, $h_{C_{3}}$, in the following manner:

$$
h_{C_{3}}=\inf \{h>0 \mid \psi \equiv 0, A=h x+C \text { is the unique solution of (1.1) }\} \text {. }
$$

$h_{C_{3}} \approx 1.7 \kappa$ is also known as DeGenne's value [15].

In this section we prove the following result:

Theorem 2.1. Let $\frac{1}{\sqrt{2}}<\kappa$ and let $\frac{1}{\sqrt{2}}<h<h_{c_{3}}$. Then there exists at least one solution $(\psi, A)$ to (1.1), for which $0<\psi<1$. 
To prove the above result we examine the initial value problem

$$
\begin{gathered}
\frac{\psi^{\prime \prime}}{\kappa^{2}}=\psi^{3}-\psi+A^{2} \psi, \\
A^{\prime \prime}=\psi^{2} A, \\
\psi^{\prime}(0)=0 \quad ; \quad \psi(0)=\psi_{0}, \\
A^{\prime}(0)=h \quad ; \quad A(0)=-\sqrt{1-\frac{1}{2} \psi_{0}^{2}},
\end{gathered}
$$

whose solution must exist in the interval $[0, x)$ for some $x>0$. For fixed $\frac{1}{\sqrt{2}}<\kappa$ and $\frac{1}{\sqrt{2}}<h$ we examine the solutions of (2.1) for $0<\psi_{0}<1$, and show that at least one of them satisfy (1.1) and $0<\psi<1$ for all $x>0$. Before proving Theorem 2.1 we need, therefore, to prove some general statements concerning any solution of (2.1).

Lemma 2.2. Let $(\psi, A)$ be a solution of (2.1) with $0<\psi_{0} \leq 1$ and $h>1 / \sqrt{2}$. If $\exists x_{0} \geq 0$ such that $\psi\left(x_{0}\right)=1$ and $\psi^{\prime}\left(x_{0}\right) \geq 0$, then $\exists x_{1}>x_{0}$ such that $\lim _{x \uparrow x_{1}} \psi=\infty$.

Proof: Suppose first that $\psi^{\prime}\left(x_{0}\right)>0$. Then, since by $(2.1 \mathrm{k}) \psi$ cannot have a local maximum where $\psi>1$ [11, we have $\psi^{\prime}>0$ for all $x>x_{0}$. Multiplying (2.1 a) by $\psi^{\prime}$ and integrating between $x_{0}$ and $x$, we obtain

$$
\left.\left[\frac{\left(\psi^{\prime}\right)^{2}}{\kappa^{2}}-\frac{1}{2} \psi^{4}+\psi^{2}\right]\right|_{x_{0}} ^{x} \geq 0 \Longrightarrow \frac{\left(\psi^{\prime}\right)^{2}}{\kappa^{2}} \geq \frac{1}{2}\left(1-\psi^{2}\right)^{2} \forall x \geq x_{0}
$$

which yields

$$
\left|\frac{\psi-1}{\psi+1}\right| \geq C e^{\sqrt{2} \kappa x} \text {. }
$$

Consequently, when the right-hand-side of the above inequality becomes greater than unity, $\psi$ can no longer exist.

Let then $\psi^{\prime}\left(x_{0}\right)=0$. If $A^{2}\left(x_{0}\right)>0$ then $\psi^{\prime \prime}\left(x_{0}\right)>0$ and hence $\psi^{\prime}>0$ for all $x>x_{0}$. We can then obtain (2.2) by repeating the same steps as before. Finally, if $A\left(x_{0}\right)=0$ we obtain from the Hamiltonian relation

$$
\frac{\left(\psi^{\prime}\right)^{2}}{\kappa^{2}}+\left(A^{\prime}\right)^{2}-\frac{1}{2} \psi^{4}+\psi^{2}-\psi^{2} A^{2}=h^{2}
$$

that $\left(A^{\prime}\right)^{2}\left(x_{0}\right)=h^{2}-\frac{1}{2}>0$. Hence, $\psi^{(3)}\left(x_{0}\right)=0$ but $\psi^{(4)}\left(x_{0}\right)=2 h^{2}-1>0$, and therefore, $\psi^{\prime}>0$ for all $x>x_{0}$ and we can proceed as before.

We note that the same result holds for $(-\psi, A)$, or equivalently, if $\exists x_{0} \geq 0$ such that $\psi\left(x_{0}\right)=-1$ and $\psi^{\prime}\left(x_{0}\right) \leq 0$, then $\exists x_{1}>x_{0}$ such that $\lim _{x \uparrow x_{1}} \psi=-\infty$.

Lemma 2.3. Any solution $(\psi, A)$ of (2.1) which exists in $[0, \infty)$ with $h>1 / \sqrt{2}$ must satisfy:

$$
\lim _{x \rightarrow \infty} A^{2}=\infty .
$$

Proof: By (2.1b), $A$ can have neither a non-positive minimum or a non-negative maximum (if at some point $A=A^{\prime}=0$, then $A \equiv 0$ ). Hence, if $\exists x_{0}>0$ such that $A\left(x_{0}\right)=0$, then $A^{\prime}>A^{\prime}\left(x_{0}\right)>0$ for all $x>x_{0}$, and thus, $A \rightarrow \infty$ as $x \rightarrow \infty$. If, on the 
other hand, $\exists x_{0}>0$ such that $A^{\prime}\left(x_{0}\right)=0$ and $A\left(x_{0}\right)<0$, then both $A^{\prime}$ and $A^{\prime \prime}$ must be negative for all $x<x_{0}$, and hence $A \rightarrow-\infty$ as $x \rightarrow \infty$.

Suppose now, for a contradiction, that $A^{\prime}>0$ for all $x>0$ and that $A \rightarrow A_{\infty} \leq 0$ as $x \rightarrow \infty$. Since $A^{\prime \prime}<0$ for all $x, A^{\prime} \rightarrow 0$ as $x \rightarrow \infty$, and hence $A^{\prime \prime}=\psi^{2} A \rightarrow 0$ as well. If $\psi \rightarrow 0$, then by (2.3) $\frac{\left(\psi^{\prime}\right)^{2}}{\kappa^{2}} \rightarrow h^{2}-$ a contradiction. If $A \rightarrow 0$, then there exists $x_{M}$ such that when $x>x_{M},\left(A^{\prime}\right)^{2}<\left(h^{2}-1 / 2\right) / 2$. Hence, for any $x>x_{M}$

$$
\frac{\left(\psi^{\prime}\right)^{2}}{\kappa^{2}}>\frac{1}{2} \psi^{4}-\psi^{2}+h^{2}-\frac{h^{2}-\frac{1}{2}}{2} \geq \frac{h^{2}-\frac{1}{2}}{2}
$$

and since $h>\frac{1}{\sqrt{2}}$ we must have for some $x_{0} \geq 0 \psi\left(x_{0}\right)= \pm 1$ and $\psi \psi^{\prime}\left(x_{0}\right) \geq 0$. By Lemma $2.2(\psi, A)$ cannot exist in that case for all $x>0$.

Lemma 2.4. Let $(\psi, A)$ be any solution of (2.1) that exists in $[0, \infty)$ with $h>1 / \sqrt{2}$. Then,

$$
\lim _{x \rightarrow \infty} \psi=0 .
$$

Proof: By Lemma 2.3 for sufficiently large $x$ we have

$$
\psi^{2}-1+A^{2}>0 \text {. }
$$

Hence, $\psi$ must be either convex or concave, and thus, monotone for sufficiently large $x$. Since $\psi$ is bounded by Lemma 2.2, we have $\psi \underset{x \rightarrow \infty}{\longrightarrow} \psi_{\infty}$. Hence, $\psi^{\prime \prime} \underset{x \rightarrow \infty}{\longrightarrow} 0$, and therefore $\psi_{\infty}=0$.

Proof of Theorem 2.1: We first define a pair of subsets, $S_{1}$ and $S_{2}$, of the interval $(0,1]$ in the following manner:

(1) $\psi_{0} \in S_{1} \Rightarrow$ any solution $(\psi, A)$ of (2.1) must satisfy

$$
\begin{aligned}
& \text { (a) } \exists x_{1}>0 ; \psi \underset{x \uparrow x_{1}}{\longrightarrow} \text {, } \\
& \text { (b) } \psi>0 \forall 0 \leq x<x_{1} .
\end{aligned}
$$

(2) $\psi_{0} \in S_{2} \Rightarrow$ Any solution $(\psi, A)$ of (2.1) must satisfy $\psi<0$ on some open subset of $\mathbb{R}^{+}$.

The sets $S_{1}$ and $S_{2}$ are clearly disjoint. They are also open in view of the continuity of the solution of (2.1) in $\psi_{0}$ and Lemma 2.2. It is easy to show that $S_{1}$ is non-empty: for $\psi_{0}=1$ the solution must blow up for some finite $x_{1}>0$ by Lemma 2.2 .

To show that $S_{2}$ is non-empty we consider the limit $\psi_{0} \rightarrow 0$. Denote by $\phi(x, h)$ the solution of the linearized problem

$$
\begin{aligned}
& \frac{\phi^{\prime \prime}}{\kappa^{2}}=\left[(h x-1)^{2}-1\right] \phi, \\
& \phi(0)=1 \quad ; \quad \phi^{\prime}(0)=0 .
\end{aligned}
$$

We claim that as $\psi_{0} \rightarrow 0, \psi \sim \psi_{0} \phi\left[1+O\left(\psi_{0}^{2}\right)\right]$ on any finite interval $[0, x)$. To prove this statement we first note that for $\psi_{0}=0$ the unique solution is $\psi \equiv 0, A=h x-1$. Thus, using, for instance, Theorem V.2 in [6] we obtain for some positive constant $C$ which may depend on $x$ that

$$
\|\psi\|_{L^{\infty}[0, x]} \leq C \psi_{0}
$$

Consequently, by (2.1b), we have

$$
\|A-(h x-1)\|_{L^{\infty}[0, x]} \leq C \psi_{0}^{2}
$$


( $C$ need not be the same constant as before although we keep the same notation). We can now apply Theorem V.3 in [6] to show that as $\psi_{0} \rightarrow 0$,

$$
\left\|\psi-\psi_{0} \phi\right\|_{L^{\infty}[0, x]} \leq C \psi_{0}^{3} .
$$

Hence, if $\phi$ is negative in some open subset of $\mathbb{R}^{+}$, then, for sufficiently small $\psi_{0}, \psi$ must be negative on some open subset of $\mathbb{R}^{+}$as well.

Let $h=\kappa$. Then, the solution of (2.4) is given by

$$
\phi(x, \kappa)=\left[1-\frac{\kappa}{e} \int_{0}^{x} e^{(\kappa t-1)^{2}} d t\right] e^{\frac{1}{2}\left[1-(\kappa x-1)^{2}\right]} .
$$

Obviously, $\phi \rightarrow-\infty$ as $x \rightarrow \infty$, and hence, at least for $h=\kappa, S_{2}$ is not empty, and $\exists x_{0}>0$ such that $\phi\left(x_{0}, \kappa\right)=0$. Since $\phi(x, h)$ is continuously differentiable in $h$ in view of (2.4 a), there exists a continuously differentiable function $\tilde{x}(h)$, in some neighborhood of $h=\kappa$, such that $\phi(\tilde{x}, h)=0$. This function would exist as long as $\phi^{\prime}(\tilde{x}, h) \neq 0$. Clearly, if $\phi^{\prime}(\tilde{x}, h)=0$ for some finite $\tilde{x}$, then $\phi \equiv 0$ for all $x$. Thus, $S_{2}$ may become empty only if $\exists h_{0}$ such that $\tilde{x} \underset{h \rightarrow h_{0}}{\longrightarrow} \infty$. If such $\tilde{x}$ exists, then $\phi\left(x, h_{0}\right)$ must decay exponentially fast as $x \rightarrow \infty$ (cf. Theorem 36.1 in [17]).

It is well known [15] that when $h=h_{C_{3}}$, a positive decaying solution of (2.4) exists. If we prove that for no $h<h_{C_{3}}$ there is a positive solution of (2.4) which decays at infinity, then $S_{2}$ is not empty for any $h<h_{C_{3}}$. Suppose then, for a contradiction that $\exists 0<h_{1}<h_{C_{3}}$ such that $\phi\left(x, h_{1}\right)$ is a positive solution of (2.4) which decays at infinity. Let

$$
\phi_{0}(\xi)=\phi\left(\frac{\xi}{\sqrt{\kappa h_{C_{3}}}, h_{C_{3}}}\right) \quad ; \quad \phi_{1}(\xi)=\phi\left(\frac{\xi}{\sqrt{\kappa h_{1}}}, h_{1}\right) .
$$

Then,

$$
\begin{array}{ccc}
\phi_{0}^{\prime \prime}=\left[\xi^{2}-2 \alpha_{0} \xi\right] \phi_{0} & \alpha_{0}=\sqrt{\frac{\kappa}{h_{C_{3}}}} \\
\phi_{1}^{\prime \prime}=\left[\xi^{2}-2 \alpha_{1} \xi\right] \phi_{1} \quad \alpha_{1} & =\sqrt{\frac{\kappa}{h_{1}}} \\
\phi_{0}(0)=1 \quad ; & \phi_{0}^{\prime}(0)=0 \\
\phi_{1}(0)=1 ; & \phi_{1}^{\prime}(0)=0 .
\end{array}
$$

We now subtract the product of $(2.5 \mathrm{~b})$ by $\phi_{0}$ from the product of $(2.5 \mathrm{a})$ by $\phi_{1}$ and integrate over $\mathbb{R}^{+}$to obtain

$$
\left(\alpha_{0}-\alpha_{1}\right) \int_{0}^{\infty} \phi_{0} \phi_{1} \xi d \xi=0
$$

and hence $\phi_{1}$ cannot be positive on $\mathbb{R}^{+}$.

Thus $S_{1}$ and $S_{2}$ are disjoint, open and non-empty for $\frac{1}{\sqrt{2}}<h<h_{c_{3}}$. Since $(0,1]$ is connected, $S_{1} \bigcup S_{2} \neq(0,1]$. Hence, there exists $\psi_{0} \in(0,1]$ whose corresponding solution $(\psi, A)$ of (2.1) in $\mathbb{R}^{+}$must satisfy by Lemma $2.20<\psi<1$ for all $x \geq 0$.

By Lemma 2.4 $\psi \underset{x \rightarrow \infty}{\longrightarrow} 0$, and in view of the convexity of $\psi, \psi^{\prime}$ and $\psi^{\prime \prime}$ must decay at infinity as well. Hence, by (2.17a) $\psi A^{2} \underset{x \rightarrow \infty}{\longrightarrow} 0$ and therefore, from the Hamiltonian 
relation (2.3) we obtain

$$
\lim _{x \rightarrow \infty} A^{\prime}= \pm h
$$

To complete the proof of the theorem it remains necessary to show that $A^{\prime}$ tends to $h$ and not to $-h$. Suppose then, for a contradiction that $A^{\prime} \rightarrow-h$. Clearly, $A$ must have a negative maximum in that case at some point $x=x_{1}>0$. At $x=x_{1}$ we have by (2.3)

$$
\left.\left[\frac{\left(\psi^{\prime}\right)^{2}}{\kappa^{2}}-\frac{1}{2} \psi^{4}+\psi^{2}\right]\right|_{x_{1}}>h^{2} .
$$

Suppose first that $\psi^{\prime}\left(x_{1}\right)>0$. Consequently, by (2.2) the above inequality must hold for any interval $\left[x_{1}, x\right]$ on which $\psi^{\prime}>0$. However, from (2.6) follows $\psi^{\prime}\left(x_{1}\right)>\kappa \sqrt{h^{2}-1 / 2}$, and hence by bootstrapping we obtain $\psi^{\prime}>0$ for all $x>x_{1}$, which clearly contradicts Lemma 2.4.

If, on the other hand $\psi^{\prime}\left(x_{1}\right)<0$, then in some interval $\left(x_{0}, x_{1}\right]$ in which $\psi^{\prime}<0$, we have

$$
\frac{d}{d x}\left[\frac{\left(\psi^{\prime}\right)^{2}}{\kappa^{2}}-\frac{1}{2} \psi^{4}+\psi^{2}\right]<0 .
$$

Since $\psi^{\prime \prime}(0)>0$ there must be such a point $x=x_{0}$, where $\psi^{\prime}=0$. Clearly,

$$
\left.\left[\frac{\left(\psi^{\prime}\right)^{2}}{\kappa^{2}}-\frac{1}{2} \psi^{4}+\psi^{2}\right]\right|_{x_{0}}<\frac{1}{2},
$$

which contradicts (2.6) and (2.7). Finally, if $A^{\prime}\left(x_{1}\right)=\psi^{\prime}\left(x_{1}\right)=0$, then by (2.3)

$$
\psi^{2}-\frac{1}{2} \psi^{4}>h^{2}
$$

which is once again a contradiction with $h^{2}>1 / 2$.

If $h>h_{c_{3}}$ the set $S_{2}$ becomes empty, and hence, the above arguments become inapplicable. It is reasonable to assume that for $h>h_{c_{3}}$ no solution can exist to (1.1). The requirement $h>1 / \sqrt{2}$ is necessary both in the proof of Lemma 2.2 and the argument showing that $A^{\prime}$ tends to $h$ and not to $-h$. For $h \leq 1 / \sqrt{2}$ it is demonstrated in the next section that no solution can exist to (1.1). We note that the results in this section are applicable to type I superconductors as well, as long as $h_{c_{3}} \approx 1.7 \kappa>1 / \sqrt{2}$, as the requirement $\kappa>1 / \sqrt{2}$ was never used per se. Nevertheless, since $h$ must be greater than $1 / \sqrt{2}$, Theorem 2.1 ceases to be valid when $1.7 \kappa \leq 1 / \sqrt{2}$.

3. Non-existence. In this section we prove the following result:

Theorem 3.1. Let $\kappa>\frac{1}{\sqrt{2}}$ and $h \leq \frac{1}{\sqrt{2}}$. Then no positive solution can exist to (1.1), except for the normal state $\psi \equiv 0, A=h x+C$.

In order to prove the theorem we first derive the asymptotic behaviour of $\psi$ as $x \rightarrow \infty$.

Lemma 3.2. Let $(\psi, A)$ be a solution of (1.1). Then

$$
\begin{gathered}
\psi=\chi(x) U\left(-\frac{\kappa}{2 h}, \xi\right) \\
A \sim h x-a+O\left(e^{-\alpha x^{2}}\right) \text { as } x \rightarrow \infty
\end{gathered}
$$


wherein $U$ is a parabolic cylinder function of the first kind, $a$ and $\alpha$ are some positive numbers,

$$
\xi=\sqrt{2 \kappa h}\left(x-\frac{a}{h}\right)
$$

and $\chi$ satisfies

$$
\chi(x) \sim \chi_{0}+O\left(e^{-\alpha x^{2}}\right) .
$$

Proof: We first note that (3.1b) has already been proved in [11]. Following [1] we then multiply $\left(1.1^{2}\right)$ by $\psi^{\prime}$ and integrate over $(x, \infty)$ to obtain, for sufficiently large $x$,

$$
-\frac{1}{2} \psi^{\prime 2}=\kappa^{2} \int_{x}^{\infty} \psi \psi^{\prime}\left(\psi^{2}+A^{2}-1\right) d t>-\frac{1}{2} K x^{2} \psi^{2},
$$

where $K$ is any positive number smaller than $\kappa h$. Hence, taking into account that $\psi^{\prime}<0$ for sufficiently large $x$ we must have

$$
\psi \leq e^{-K x^{2}}
$$

for any $0<K<\kappa h / 2$. By (1.1. b) we obtain

$$
A \sim h x-a+O\left(e^{-K x^{2}}\right) \text { as } x \rightarrow \infty
$$

for the same values of $K$.

It is not difficult to show using variation of parameters, in view of (3.2), that any decaying solution of $(1.1 \mathrm{a})$ can be written in the form

$$
\psi=\chi(x) U\left(-\frac{\kappa}{2 h}, \xi\right)
$$

where

$$
\chi^{\prime}(x)=-\frac{\kappa^{2}}{U^{2}\left(-\frac{\kappa}{2 h}, \xi(x)\right)} \int_{x}^{\infty}\left[A^{2}-(h x-a)^{2}+\psi^{2}\right] \psi(t) U\left(-\frac{\kappa}{2 h}, \xi(t)\right) d t .
$$

Since for sufficiently large $x, U$ is monotonically decreasing, we obtain by (3.2)

$$
\left|\chi^{\prime}(x)\right| \leq \frac{e^{-2 K x^{2}}}{U\left(-\frac{\kappa}{2 h}, \xi(x)\right)} .
$$

From the well-known asymptotic behaviour of $U$ [1] we obtain $U \sim O\left(e^{-\kappa h x^{2} / 2}\right)$. Consequently, picking $K$ which is sufficiently close to $\kappa h / 2$ yields

$$
\left|\chi^{\prime}(x)\right| \leq \exp \left\{-\frac{1}{4} \kappa h x^{2}\right\}
$$

from which (3.1) immediately follows.

Proof of Theorem 3.1. Suppose for a contradiction that a solution $(\psi, A)$ to (1.1) does exist. Following [10] we define the functions

$$
\begin{gathered}
F=\sqrt{2} A^{\prime}-1+\psi^{2} \\
G=\frac{\psi^{\prime}}{\kappa}+\psi A .
\end{gathered}
$$


Using (3.2 b) and the asymptotic behaviour of $U$ as $x \rightarrow \infty$ yields (cf. also [3])

$$
\psi \sim C \exp \left\{-\frac{1}{2} \kappa h x^{2}+\kappa a x-\frac{1}{2} \frac{h-\kappa}{h} \log x+\right\}\left\{1+O\left(\frac{1}{x^{2}}\right)\right\} .
$$

Hence, as $x \rightarrow \infty$

$$
G \sim\left(-\frac{1}{2} \frac{h-\kappa}{\kappa h x}+O\left(x^{-2}\right)\right) \psi .
$$

In the present case ( $h \leq 1 / \sqrt{2}<\kappa)$; therefore, $G$ becomes positive for sufficiently large $x$. Yet, $G(0)<0$ since $A(0)$ must be negative (otherwise $\psi^{\prime \prime}$ would be monotone increasing for all $x$ ). Denote then by $x_{1}$ the last zero of $G$ in $\mathbb{R}^{+}$. Clearly, for $x>x_{1}, G$ must be positive. Hence,

$$
G^{\prime}\left(x_{1}\right) \geq 0 .
$$

However, it is easy to show [10 that

$$
G^{\prime}<\kappa(\psi F+A G) .
$$

Furthermore, writing the Hamiltonian relation (2.3) in the form

$$
F\left(\psi^{2}-1-\sqrt{2} A^{\prime}\right)-2 G\left(\frac{\psi^{\prime}}{\kappa}-\psi A\right)=1-2 h^{2}
$$

it can be easily seen that for $h \leq 1 / \sqrt{2}, F\left(x_{1}\right) \leq 0$, which together with (3.7) contradicts (3.6).

We note that the above arguments are inapplicable for type I superconductors. We expect that solutions would cease to exist for $h \leq \kappa$, but the above arguments do not support this hypothesis [2].

4. Properties as $h \downarrow 1 / \sqrt{2}$. In this section we investigate the properties of the solutions of (1.1) in the limit $h \downarrow 1 / \sqrt{2}$. To this end we need first the following lemma which summarizes a few auxiliary results.

Lemma 4.1. Any positive solution of (1.1) must satisfy

$$
\begin{aligned}
& A^{\prime}>0, \\
& 0<\psi<1, \\
& -1<A(0)<-1 / \sqrt{2} .
\end{aligned}
$$

Furthermore, $\psi$ has exactly one local maximum in $\mathbb{R}^{+}$at some point $x_{0}>0$.

Proof: For the proof of (4.17) see the proof of Lemma 2.3 Lemma 2.2 demonstrates that $\psi<1$. The proof of (4.1k) follows directly from (2.1f).

To prove the last statement (that $\psi$ has a single maximum), let $(\psi, A)$ be a solution of (1.1) for $\kappa>1 / \sqrt{2}$. It is not difficult to show that $\psi$ has exactly one maximum point at some $x=x_{0}>0$. If it has a minimum at $x=x_{1}>x_{0}$, then

$$
\left.\left(\psi^{2}+A^{2}-1\right)\right|_{x_{1}} \geq 0 \geq\left.\left(\psi^{2}+A^{2}-1\right)\right|_{x_{0}}
$$

and since $\psi\left(x_{1}\right)<\psi\left(x_{0}\right)$, we must have $A^{2}\left(x_{1}\right)>A^{2}\left(x_{0}\right)$. As $A\left(x_{1}\right)>A\left(x_{0}\right)$, we must have $A\left(x_{1}\right)>0$. However, since $\psi^{\prime \prime}\left(x_{1}\right) \geq 0$, and since $\left(\psi^{2}+A^{2}-1\right)^{\prime}>0$ as long 
as $\psi^{\prime} \geq 0$, we must have, by bootstrapping, $\psi^{\prime}>0$ for all $x>x_{1}$, which is clearly a contradiction.

In the following we investigate the asymptotic behaviour of $\psi\left(x_{0}\right), A\left(x_{0}\right)$ and $x_{0}$ in the limit $h \downarrow 1 / \sqrt{2}$. We prove the following result:

TheOrem 4.2. Let $\left\{h_{k}\right\}_{k=1}^{\infty}$ tend to $\frac{1}{\sqrt{2}}^{+}$. Denote by $\left(\psi_{k}, A_{k}\right)$ a positive solution of (1.1) with $h=h_{k}$ and $\psi_{k}(0)>0$. Then,

(1) $\left\{\left(\psi_{k}, A_{k}\right)\right\}_{k=1}^{\infty}$ contains a converging subsequence in $C^{m}[0, x]$ for any $x>0$, $m>0$.

(2) Let $\left(\psi_{k}, A_{k}\right) \rightarrow\left(\psi_{\infty}, A_{\infty}\right)$ uniformly on any finite interval $[0, x]$. Then, $\left(\psi_{\infty}, A_{\infty}\right)$ is a solution of the problem for (cf. [8])

$$
\begin{gathered}
\frac{\psi^{\prime \prime}}{\kappa^{2}}=\psi^{3}-\psi+A^{2} \psi, \\
A^{\prime \prime}=\psi^{2} A, \\
\psi^{\prime}(0)=0 \lim _{x \rightarrow \infty} \psi=1, \\
A^{\prime}(0)=1 / \sqrt{2} \lim _{x \rightarrow \infty} A=0 .
\end{gathered}
$$

We note that the proof of the theorem must include a proof of existence of solutions to 4.2 .

To prove the theorem we first show that $\psi\left(x_{0}\right) \rightarrow 1$ and $A\left(x_{0}\right) \rightarrow 0$ as $h \downarrow 1 / \sqrt{2}$.

Lemma 4.3. Let $(\psi, A)$ be a positive solution of (1.1) for $h<\kappa$. Let $x_{0}$ be the maximum point of $\psi$, and let $\psi_{m} \stackrel{\text { def }}{=} \psi\left(x_{0}\right), A_{m} \stackrel{\text { def }}{=} A\left(x_{0}\right)$. Then

$$
A_{m}^{2} \leq 1-\psi_{m}^{2} \leq \sqrt{\frac{h^{2}-\frac{1}{2}}{\kappa^{2}-\frac{1}{2}}} \quad ; \quad A^{\prime 2}\left(x_{0}\right) \leq \sqrt{\frac{h^{2}-\frac{1}{2}}{\kappa^{2}-\frac{1}{2}}}+h^{2}-\frac{1}{2} .
$$

Proof: In $\S 3$ in (3.4b) we define a function $G$ which is negative at the origin and positive for sufficiently large $x$. Hence, there is a point $x=x_{1}$ where $G\left(x_{1}\right)=0$ and $G^{\prime}\left(x_{1}\right) \geq 0$. It is easy to show, by calculating the derivative of $G$ that at this point, that

$$
\left[\psi^{2}-1+\frac{A^{\prime}}{\kappa}\right]_{x=x_{1}} \geq 0 .
$$

Let $\psi_{1}=\psi\left(x_{1}\right)$ and $A_{1}^{\prime}=A^{\prime}\left(x_{1}\right)$. By the above inequality we have

$$
\kappa^{2}\left(1-\psi_{1}^{2}\right)^{2} \leq\left(A_{1}^{\prime}\right)^{2} .
$$

By (3.2 a), 2.3) is valid for any solution of (1.1). At $x=x_{1}$, keeping in mind that $G\left(x_{1}\right)=0,(2.3)$ is expressible in the form

$$
\left(A_{1}^{\prime}\right)^{2}-\frac{1}{2}\left(1-\psi_{1}^{2}\right)^{2}=h^{2}-\frac{1}{2} .
$$

Hence,

$$
\kappa^{2}\left(1-\psi_{1}^{2}\right)^{2} \leq \frac{1}{2}\left(1-\psi_{1}^{2}\right)^{2}+h^{2}-\frac{1}{2} .
$$


Consequently, as $\psi_{1} \leq \psi_{m}$, we have

$$
\left(1-\psi_{m}^{2}\right) \leq\left(1-\psi_{1}^{2}\right) \leq \sqrt{\frac{h^{2}-\frac{1}{2}}{\kappa^{2}-\frac{1}{2}}} .
$$

Obviously, since $x=x_{0}$ is a maximum point, we must have $\psi^{\prime \prime}\left(x_{0}\right) \leq 0$ and hence,

$$
A_{m}^{2} \leq 1-\psi_{m}^{2}
$$

which proves $4.3 \mathrm{a})$.

To prove (4.3p) we use (2.1) at $x=x_{0}$ to obtain

$$
A^{\prime}\left(x_{0}\right)^{2}-\frac{1}{2}\left(1-\psi_{m}^{2}\right)^{2}=\psi_{m}^{2} A_{m}^{2}+h^{2}-\frac{1}{2}
$$

which, combined with (4.4), yields

$$
A^{\prime}\left(x_{0}\right)^{2} \leq h^{2}-\frac{1}{2} \psi_{m}^{4} .
$$

Substituting (4.3 $\mathrm{a})$ in the above proves (4.3 $\mathrm{b})$.

Next, we show that $x_{0} \rightarrow \infty$ as $h \downarrow 1 / \sqrt{2}$.

Lemma 4.4. Let $(\psi, A)$ be a positive solution of (1.1), and let $x_{0}$ denote the maximum point of $\psi$. Then

$$
x_{0}>C+\frac{1}{4} \log \frac{1}{h^{2}-\frac{1}{2}} .
$$

Proof: Denote by $\tilde{A}$ the solution of the problem

$$
\begin{aligned}
\tilde{A}^{\prime \prime}=\tilde{A} & \text { in }\left(0, x_{0}\right) \\
\tilde{A}\left(x_{0}\right)=-\left|A\left(x_{0}\right)\right| & ; \quad \tilde{A}^{\prime}\left(x_{0}\right)=A^{\prime}\left(x_{0}\right) .
\end{aligned}
$$

The solution of (4.8) is given by

$$
\tilde{A}(x)=-\left|A\left(x_{0}\right)\right| \cosh \left(x-x_{0}\right)+A^{\prime}\left(x_{0}\right) \sinh \left(x-x_{0}\right),
$$

which together with (4.3) yields the estimate

$$
-C\left[h^{2}-\frac{1}{2}\right]^{1 / 4} e^{x_{0}-x} \leq \tilde{A}<0 .
$$

In view of (1.1 b) and (4.8 a), it is not difficult to show that $\tilde{A} \leq A$ for all $x \in\left[0, x_{0}\right]$. Hence, $\tilde{A}(0)<A(0)<-1 / \sqrt{2}$, from which (4.7) easily follows.

Proof of Theorem 4.2. Since (2.3) is valid for any solution of (1.1) [in view of (3.2 $\mathrm{a}$ )] and since $A(0)$ must be negative, it is clear that any solution satisfying (1.1) must be a solution of (2.1) for some $0<\psi_{0} \leq 1$. Namely,

$$
\begin{gathered}
\frac{\psi_{k}^{\prime \prime}}{\kappa^{2}}=\psi_{k}^{3}-\psi_{k}+A_{k}^{2} \psi_{k}, \\
A_{k}^{\prime \prime}=\psi_{k}^{2} A_{k}, \\
\psi_{k}^{\prime}(0)=0 \quad ; \quad \psi_{k}(0)=\psi_{0}^{(k)}, \\
A_{k}^{\prime}(0)=h_{k} \quad ; \quad A_{k}(0)=-\sqrt{1-\frac{1}{2}\left(\psi_{0}^{(k)}\right)^{2}}
\end{gathered}
$$


where $0<\psi_{0}^{(k)}<1$. Hence, we can assume $\psi_{0}^{(k)} \rightarrow \psi_{0}^{\infty}$ or else move to an appropriate subsequence. Let $(\phi, B)$ denote the solution of

$$
\begin{gathered}
\frac{\phi^{\prime \prime}}{\kappa^{2}}=\phi^{3}-\phi+B^{2} \phi, \\
B^{\prime \prime}=\phi^{2} B \\
\phi^{\prime}(0)=0 \quad ; \quad \phi(0)=\psi_{0}^{\infty}, \\
B^{\prime}(0)=1 / \sqrt{2} \quad ; \quad B(0)=-\sqrt{1-1 / 2\left(\psi_{0}^{\infty}\right)^{2}} .
\end{gathered}
$$

By continuity of solutions with respect to initial conditions (cf. Theorem 4.1 in chapter $\mathrm{V}$ of [13]) together with the extension theorem (cf. Theorem 3.1 in chapter II of [13]), it now immediately follows that $\left(\psi_{k}, A_{k}\right) \rightarrow(\phi, B)$ in $C^{m}[0, x]$ for any $x>0$ and $m>0$.

To prove that $(\phi, B) \equiv\left(\psi_{\infty}, A_{\infty}\right)$ we first need to show that $\psi_{0}^{\infty}>0$. Suppose, for a contradiction, that $\psi_{0}^{\infty}=0$. Then, $\left(\psi_{k}, A_{k}\right)$ must tend pointwise to

$$
\psi \equiv 0 \quad ; \quad A=\frac{x}{\sqrt{2}}-1
$$

Denote by $x_{0}^{(k)}$ the maximum point of $\psi_{k}$. By Lemma $4.3 A_{k}\left(x_{0}^{(k)}\right) \rightarrow 0$ as $k \rightarrow \infty$. Furthermore, $A_{k}(x)<A_{k}\left(x_{0}^{(k)}\right)$ for all $x<x_{0}^{(k)}$, and since by Lemma $4.4 x_{0}^{(k)} \rightarrow \infty$ as $k \rightarrow \infty, A_{k} \nrightarrow A$ yielding $\psi_{0}^{\infty}>0$.

It remains necessary to show only that $(\phi, B)$ satisfies $4.2 \mathrm{~d})$ and $(4.2 \mathrm{f})$. It has already been demonstrated that for $x<x_{0}^{(k)}$, we have

$$
\psi_{k}(x)<\psi_{k}\left(x_{0}^{(k)}\right) ; A_{k}(x)<A_{k}\left(x_{0}^{(k)}\right) ; \psi_{k}^{\prime}(x)>0 ; A_{k}^{\prime}(x)<0 .
$$

Hence, since $x_{0}^{(k)} \rightarrow \infty$ we have $\phi^{\prime} \geq 0$ and $B^{\prime} \geq 0$ for all $x>0$. Furthermore, by Lemma 4.3 we have $\phi \leq 1$ and $B \leq 0$ for all $x>0$. Thus, both $\phi$ and $B$ must tend to constants, which we respectively denote by $\phi_{\infty}$ and $B_{\infty}$, at infinity. By (4.9a, $\left.\mathrm{b}\right)$ we must then have $\phi^{\prime \prime} \rightarrow 0$ and $B^{\prime \prime} \rightarrow 0$ and since $\phi_{\infty}>\psi_{0}^{\infty}>0$, we must have $\phi_{\infty}=1, B_{\infty}=0$.

5. Conclusion. We have demonstrated for type II superconductors that

(1) Positive solutions $(\psi>0)$ exist whenever $1 / \sqrt{2}<h<h_{c_{3}} \approx 1.7 \kappa$;

(2) No positive solutions can exist for $h \leq 1 / \sqrt{2}$;

(3) As $h \downarrow 1 / \sqrt{2}$ the limit of any converging subsequence satisfies $A=0, \psi=1$ at infinity.

For type I superconductors only the results (1) and (3) are proved, and only for the case $1.7 \kappa>1 / \sqrt{2}$. We note that since the normal state is known to be unstable for $h<1.7 \kappa$, result (2) does not mean that the normal state would prevail but rather that any solution satisfying the normal state condition at infinity must be unstable (or time-dependent). Result (3) indicates that steady solutions must satisfy the perfectly superconducting state at infinity.

The above results are in agreement with Conjecture 4.8 in [2], which discusses the existence of asymmetric solutions of $(\mathbf{1 . 1}, \mathrm{b})$ on the interval $[-a, a]$ in the limit $a \rightarrow \infty$. There are two types of asymmetric solutions according to this conjecture: one that can be approximated by the solution of (4.2) with $A^{\prime}(0)$ not necessarily equal to $1 / \sqrt{2}$, and one that can be approximated by the solution of (1.1). We note that for the linearized 
equations it was proved [7] that the solutions in the interval $[-a, a]$ satisfying $\psi^{\prime}( \pm a)=0$ tend to the solution in $\mathbb{R}^{+}$as $a \rightarrow \infty$.

It should be mentioned that the solution on the real line, which represents one of the symmetric modes on $[-a, a]$ as $a \rightarrow \infty$, does not exist for $h \leq 1 / \sqrt{2}$ for type II superconductors [3. Furthermore, formal asymptotic expansion for $\kappa \approx 1 / \sqrt{2}$ shows that as $h \rightarrow 1 / \sqrt{2}$ the solution tends pointwise to $\psi \equiv 1 A \equiv 0$. Both results resemble the results in $\S 3$ and $\S 4$. Finally, it is reasonable to believe that for some $h<\kappa$ the solutions of (1.1) become unstable in two dimensions. It is expected that periodic solutions in the form of Abrikosov's lattices would emerge away from the boundaries for sufficiently low field.

\section{REFERENCES}

[1] M. Abramowitz and I. A. Stegun, Handbook of mathematical functions, Dover, 1972.

[2] A. Aftalion and W. C. Troy, On the solution of the one-dimensional Ginzburg-Landau equations of superconductivity, Phys. D 132 (1999), 214-232. MR1705706 (2000h:82096)

[3] Y. Almog, Asymptotic analysis of the one-dimensional Ginzburg-Landau equations near self-duality, Quart. Appl. Math. 57 (1999), 355-367. MR1686194 (2000c:35214)

[4] P. Bauman, D. Philips, and Q. Tang, Stable nucleation for the Ginzburg-Landau system with an applied magnetic field, Arch. Rat. Mech. Anal. 142 (1998), 1-43. MR.1629119 (99g:58040)

[5] A. Bernoff and P. Sternberg, Onset of superconductivity in decreasing fields for general domains, J. Math. Phys. 39 (1998), 1272-1284. MR1608449 (99a:82099)

[6] G. Birkhoff and G. Rota, Ordinary differential equations, Ginn and Company, 1962. MR0138810 $(25: 2253)$

[7] C. Bolley and B. Helffer, Rigorous results on Ginzburg-Landau models in a film submitted to exterior parallel magnetic field i, Nonlinear Studies 3 (1996), 1-29. MR1396033 (97e:82050)

[8] The Ginzburg-Landau equations in a semi-infinite superconducting film in the large $\kappa$ limit, EJAM 8 (1997), 347-367. MF 1471597 (98k:82200)

[9] S. J. Chapman, Nucleation of superconductivity in decreasing fields I, EJAM 5 (1994), 449-468. MR.1309734 (95m:82119)

[10] _ Asymptotic analysis of the Ginzburg-Landau model of superconductivity: Reduction to a free boundary model, Quart. Appl. Math. 53 (1995), 601-627. MR1359498 (96j:82049)

[11] S. J. Chapman, S. D. Howison, J. B. Mcleod, and J. R. Ockendon, Normal/superconducting transition in Landau-Ginzburg theory, Proc. Roy. Soc. Edinburgh 119A (1991), 117-124. MR1130600 (92m:82149)

[12] M. del Pino, P. L. Felmer, and P. Sternberg, Boundary concentration for eigenvalue problems related to the onset of the superconductivity, preprint. MR,1776839 (2001k:35231)

[13] P. Hartman, Ordinary differential equations, SIAM, 2002. MR.1929104 (2003h:34001)

[14] K. Lu and X.B. Pan, Eigenvalue problems of Ginzburg-Landau operator in bounded domains, J. Math. Phys. 50 (1999), 2647-2670. MR1694223 (2001c:35167)

[15] D. Saint-James and P.G. de Gennes, Onset of superconductivity in decreasing fields, Phys. Let. 7 (1963), 306-308.

[16] S. Serfaty, Stable configurations in superconductivity: Uniqueness, multiplicity, and vortexnucleation, Arch. Ration. Mech Anal. 149 (1999), 329-365. MR.1731999|(2001h:82114)

[17] Y. Sibuya, Global theory of a second order linear differential equation with a polynomial coefficient, North Holland, 1975. MR 0486867|(58:6561) 